\title{
Convergence Properties of Dynamic Agents Consensus Networks with Broken Links
}

\author{
S. Di Cairano, A. Pasini, A. Bemporad, R.M. Murray
}

\begin{abstract}
Convergence properties of distributed consensus protocols on networks of dynamical agents have been analyzed by combinations of algebraic graph theory and control theory tools under certain assumptions, such as strong connectivity. Strong connectivity can be regarded as the requirement that the information of each agent propagates to all the others, possibly with intermediate steps and manipulations. However, because of network failures or malicious attacks, it is possible that this assumption no longer holds, so that some agents are only receiving or only transmitting information from other subsets of agents. In this case, strong connectivity is replaced by weak connectivity. We analyze the convergence properties of distributed consensus on directed graphs with weakly connected components. We show conditions for which the agreement is reached, and, for the cases in which such conditions do not hold, we provide bounds on the residual disagreement.
\end{abstract}

\section{INTRODUCTION}

Distributed consensus algorithms [1], [2] are applied to groups of agents in order to have them reaching an agreement. Distributed consensus algorithms can be seen as protocols operating within networks of dynamical systems [3][6]. In particular, average consensus is useful for distributed estimation of static and dynamic quantities [7].

In distributed consensus each agent recursively solves a local problem with smaller size of input data with respect to the global problem [2], requiring information only from its "neighbors", a subset of the other agents. Given an agent, its neighbors are the agents that are close to it, in a network topological sense. When an agent has solved its local problem, it updates its state, obtains new information from the neighbors, and solves a new problem. The main advantage of the consensus protocols is that the operations are distributed among the agents, while the drawback is that communications among agents are required at each step of the algorithm. In distributed consensus under certain assumptions each agent converges to a value which is equal to the convergence values of all the other agents. For instance, in average consensus the state of each agent converges to the average of the initial values of the agents within the network. The assumptions that guarantee convergence are especially

S. Di Cairano and A. Bemporad are with Dipartimento di Ingegneria dell'Informazione, Università di Siena, dicairano, bemporadedii.unisi.it

A. Pasini is with Dipartimento di Matematica, Università di Siena pasini@unisi.it

R.M. Murray is in the Division of Engineering and Applied Science at the California Institute of Technology murray acds. caltech.edu

S. Di Cairano and A. Bemporad were partially supported by the European Community through the Network of Excellence HYCON, FP6-IST-511368.

S. Di Cairano and R.M. Murray were partially supported by Boeing through the project Model-Based Design and Qualification of Complex Systems. related to the topological properties of the network, and in particular to its connectivity.

The capability of convergence properties to survive to network modifications gives a measure of algorithm robustness [8]. Robustness is a crucial point in a distributed protocol, since in general the agents interact with a changing environment [2] that affects the computation. As regards the consensus protocols, in [4] the case of switching network topology and the effects of communication delay have been studied. In [8], the robustness of the consensus protocol with respect to connectivity losses has been characterized in terms of the number of agents that must fail for the network to become disconnected. In such a case, two or more autonomous groups of agents that do not interact with each other are created. This is certainly a critical situation, because the cooperation is broken, but, on the other hand, the smaller networks operate as the former, single network, thus ensuring that the properties still hold within the subgroups.

In this paper we analyze the situation in which the strong connectivity of a network is broken, and the new network is composed of one or more weakly connected components. The subgroups of agents are still connected, but among them the information flows in a single direction (some subgroups are only transmitting to/receiving from some others). In this case the properties that require strong connectivity neither hold in the whole network, nor restrictedly to the weakly connected components. This condition is relatively common in real systems such as sensor networks, due to events such as node failures, or failures that affect only either the transmitter or the receiver of a node, radio channels interruptions (for instance due to shadowing), repeated packet losses due to congestion or interference, and so on. Such a situation may be also maliciously generated by an external attack to a node.

The paper is structured as follows. In Section II we review the main results of distributed consensus on directed graphs, and in Section III we analyze the graph theoretic and algebraic properties of graphs with weakly connected components. We use these results in combination with control theory in Section IV to analyze asymptotic convergence of distributed consensus on networks with weakly connected components. In Section V we show simulation results of a simple case study to prove the validity of the theoretical results. Some conclusions are summarized in Section VI.

\section{A. Notation}

$\mathbb{R}$ and $\mathbb{C}$ are the set of real and complex numbers, respectively. We indicate by $\mathbf{0}$ and $\mathbf{1}$ matrices and vectors of appropriate dimensions entirely composed of 0 and 1 , respec- 
tively. Given a matrix $T, \operatorname{ker}(T)$ indicates its kernel, and $t_{i j}$ indicates the element at row $i$ and column $j \cdot \operatorname{diag}(a, b, \ldots)$ is a diagonal matrix whose entries on the diagonal are $a, b$, and so on. When $x$ is a vector, $x_{i}$ indicates the $i^{\text {th }}$ component (or coordinate) of the vector, and the relational operators when used between vectors are intended componentwise. The operators $\operatorname{Re}, \operatorname{Im}: \mathbb{C} \rightarrow \mathbb{R}$ extract the real and the imaginary part of a complex number, respectively. The operator $\backslash$ denotes difference between sets, and $|\cdot|$ the cardinality of a set.

\section{Preliminaries on Graphs AND Consensus PROTOCOLS}

We refer to the consensus protocol presented in [4], where the authors consider a directed graph (digraph) $\Gamma=$ $(\mathcal{V}, \mathcal{E}, A)$, with vertices (or nodes) $\mathcal{V}=\left\{v_{1}, \ldots, v_{n}\right\}$, and directed edges $\mathcal{E} \subseteq \mathcal{V} \times \mathcal{V}$, where an edge is denoted by $e_{i j}=\left(v_{i}, v_{j}\right)$. The matrix $A$ is the weighted adjacency matrix of the graph, where a positive value $a_{i j}>0$ is associated to each existing edge $e_{i j} \in \mathcal{E}$, and all the other entries are set to 0 . We assume that the graph has no loops of length 1 , and as a consequence $a_{i i}=0, i=1, \ldots, n$. The neighbor set of a node $v_{i}$ is $\mathcal{N}_{i}=\left\{v_{j} \in \mathcal{V}: e_{i j} \in \mathcal{E}\right\}$, and the nodes $v_{j} \in \mathcal{N}_{i}$ are referred to as neighbors of $v_{i}$.

Definition 1: A digraph is strongly connected if and only if each couple of distinct nodes $\left(v_{i}, v_{j}\right), i \neq j$ is connected with a directed path, a path that follows the direction of the edges. A digraph is weakly connected if each couple of distinct nodes $\left(v_{i}, v_{j}\right), i \neq j$ is connected with a path that does not account for the direction of the edges. A digraph is componentwise weakly connected if the connected components $^{1}$ are weakly connected.

Each node $v_{i}$ is a dynamical agent with state $x_{i} \in \mathbb{R}$ and integral dynamics

$$
\dot{x}_{i}(t)=u_{i}(t)
$$

and the distributed consensus protocol in [4] defines

$$
u_{i}(t)=\sum_{v_{j} \in \mathcal{N}_{i}} a_{i j}\left(x_{j}(t)-x_{i}(t)\right) .
$$

In this protocol an edge $e_{i j}$ indicates that the state of agent $j$ is influenced by the state of agent $i$, and hence that there is information flow from $v_{j}$ to $v_{i}$. We will use the terms agent, node, and vertex, interchangeably.

Given dynamics (1) and protocol (2), the agents dynamics are described by

$$
\dot{x}(t)=-L x(t)
$$

where $L$ is the graph Laplacian matrix [9], $\ell_{i j}=$ $\sum_{h=1, h \neq i}^{n} a_{i h}$, if $j=i, \ell_{i j}=-a_{i j}$, if $j \neq i$. Given a node $v_{i} \in \mathcal{V}, \operatorname{in}\left(v_{i}\right)=\sum_{j} a_{j i}$ and out $\left(v_{i}\right)=\sum_{j} a_{i j}$ are called indegree and out-degree of $v_{i}$, respectively. Thus, $L=\Delta-A$, where $\Delta=\operatorname{diag}\left(\operatorname{out}\left(v_{1}\right), \ldots, \operatorname{out}\left(v_{n}\right)\right)$ is a diagonal matrix with the node out-degrees on the diagonal.

The following results are discussed in [10], [11] for undirected graphs, and in [4] for digraphs.

\footnotetext{
${ }^{1}$ The connected components of a directed graph are its maximal connected subgraphs.
}

Result 1: Given a network of $n$ agents and the corresponding Laplacian matrix $L, \operatorname{rank}(L) \leq(n-1)$ and the vector 1 is a right eigenvector of $L$ corresponding to the eigenvalue $\lambda=0$. If the graph described by $L$ is strongly connected, $\operatorname{rank}(L)=n-1$. The eigenvalues of $L$ are located in the complex plane in the region $\{x \in \mathbb{C}: \operatorname{Re}(x) \geq 0,|\operatorname{Im}(x)| \leq$ $\rho$, where $\rho=\max _{i} \sum_{j}\left|\ell_{i j}\right|$.

Result 2: Given a strongly connected network of agents, agent dynamics (1) and consensus protocol (2), the state vector $x$ converges to a consensus, i.e., $\lim _{t \rightarrow \infty} x_{i}(t)=\bar{x}$, $i=1, \ldots, n$. Let $\alpha \neq \mathbf{0}$ be a nonnegative left eigenvector of $L$ relative to $\lambda=0$, the group decision value is $\bar{x}=$ $\frac{\sum_{i=1}^{n} \alpha_{i} x_{i}(0)}{\sum_{i=1}^{n} \alpha_{i}}$. If the network is balanced, i.e. $\forall v_{i} \in \mathcal{V}$, $\operatorname{in}\left(v_{i}\right)=\operatorname{out}\left(v_{i}\right)$, then $\alpha=\mathbf{1}, \bar{x}=\frac{1}{n} \sum_{i=1}^{n} x_{i}(0)$, hence the network converges to the average consensus.

The previous definitions and results are extended to the case of discrete-time agents dynamics

$$
x_{i}(t+1)=x_{i}(t)+\varepsilon u_{i}(t),
$$

provided that $0<\varepsilon<\frac{1}{\max _{i} \ell_{i i}}$. Dynamics (3) together with protocol (2) result in the closed-loop discrete-time dynamics

$$
x(t+1)=(I-\varepsilon L) x(t) .
$$

\section{Distributed CONSENSUS With BROKEN LinKS}

We analyze consensus protocols where strong connectivity does not hold. When strong connectivity is lost, the information does not propagate uniformly. In particular there exists at least one ordered couple of nodes $\left(v_{i}, v_{j}\right)$ that is not connected with a directed path, meaning that there is no information flow from $v_{j}$ to $v_{i}$. When the network becomes componentwise weakly connected, there is no straightforward way to extend the properties recalled in Section II.

\section{A. Authorities and Connectivity}

In componentwise weakly connected networks the agents play different roles depending on their connectivity. The following definitions identify the different roles.

Definition 2: Given $\Gamma(\mathcal{V}, \mathcal{E}, A), \mathcal{X} \subseteq \mathcal{V}$ is a stable set if does not exist any edge $e_{i j} \in \mathcal{E}$ such that $v_{i} \in \mathcal{X}, v_{j} \in \mathcal{V} \backslash \mathcal{X}$. A stable set $\mathcal{X}$ is minimal if does not exist any stable set $\mathcal{Y}$, such that $\mathcal{Y} \subset \mathcal{X}$.

Referring to consensus protocol (2), where an edge $e_{i j}$ indicates that the agent associated to $v_{i}$ is influenced by the agent associated to $v_{j}$, a stable set $\mathcal{X}$ is a set which is not influenced by any external agent. A stable set is minimal if by removing any node the remaining set is not stable. The following stable sets properties hold. The proofs are immediate and omitted here for brevity.

Lemma 1: Each stable set contains minimal stable sets. The intersection of stable sets is stable. Two minimal stable sets are always disjoint.

Definition 3: Given $\mathcal{Y} \subseteq \mathcal{V}$, the subgraph induced by $\mathcal{Y}$ on $\Gamma(\mathcal{V}, \mathcal{E}, A)$ is $\Gamma\left(\mathcal{Y}, \mathcal{E}_{\mathcal{Y}}, A_{\mathcal{Y}}\right)$, where $\mathcal{E}_{\mathcal{Y}}=\left\{e_{i j} \in \mathcal{E}: v_{i} \in\right.$ $\left.\mathcal{Y}, v_{j} \in \mathcal{Y}\right\}$, and $A_{\mathcal{Y}}$ is built accordingly from $A$.

Lemma 2: Given $\Gamma(\mathcal{V}, \mathcal{E}, A)$, let $\mathcal{X} \in \mathcal{V}$ be a minimal stable set. The subgraph induced by $\mathcal{X}$ on $\Gamma$ is strongly connected. 


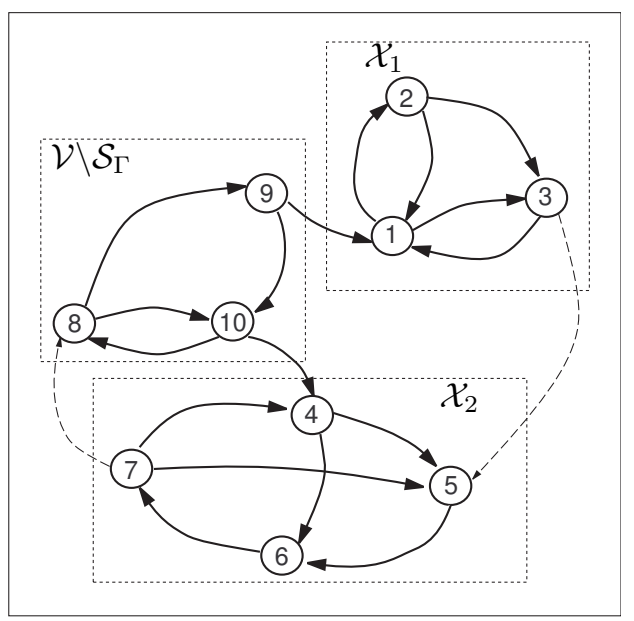

Fig. 1. When the dashed links are removed the strongly connected network becomes weakly connected with authorities $\mathcal{X}_{1}$ and $\mathcal{X}_{2}$.

Proof: Let $\Gamma\left(v_{i}\right) \subseteq \mathcal{V}$ be the set of nodes reachable from $v_{i}$ by the edges of $\Gamma$. For any $v_{i} \in \mathcal{V}, \Gamma\left(v_{i}\right)$ is stable, otherwise there would exist $\mathcal{J} \supset \Gamma\left(v_{i}\right)$ whose nodes are all reachable from $v_{i}$, which would contradict the definition of $\Gamma\left(v_{i}\right)$. Since $v_{i} \in \mathcal{X}$, and $\mathcal{X}$ is stable, $\Gamma\left(v_{i}\right) \subseteq \mathcal{X}$. But $\mathcal{X}$ is minimal, hence $\Gamma\left(v_{i}\right)=\mathcal{X}$. Thus, since the previous holds $\forall v_{i} \in \mathcal{X}, \mathcal{X}$ is strongly connected.

Definition 4: Given $\Gamma(\mathcal{V}, \mathcal{E}, A)$, we call authorities the minimal stable sets $\mathcal{X}_{i}$ of $\Gamma$, and $\mathcal{S}_{\Gamma} \triangleq \bigcup_{i} \mathcal{X}_{i}$.

We use the term authority from the viewpoint of the consensus algorithm, since the authorities are the groups of agents that base their decisions on themselves only, without being influenced by any external agent, and may be able to influence other agents. As a consequence of Lemmas 1 and 2, if $\mathcal{V}=\mathcal{S}_{\Gamma}, \Gamma$ is either strongly connected or disconnected, hence $\Gamma$ is strongly connected if and only if it is the only stable set. The following properties, whose proofs are simple and omitted here for brevity, hold.

Lemma 3: Given $\Gamma(\mathcal{V}, \mathcal{E}, A), \mathcal{V} \backslash \mathcal{S}_{\Gamma}$ does not contain any stable set. From any $v_{i} \in \mathcal{V} \backslash \mathcal{S}_{\Gamma}$, there exist a path to $\mathcal{S}_{\Gamma}$.

Figure 1 shows an example of a weakly connected graph. When both the solid and the dashed links are considered, the network is strongly connected, that is, there is only one authority, and $\mathcal{V}=\mathcal{S}_{\Gamma}$. When the dashed links are removed the network becomes weakly connected with two authorities $\mathcal{X}_{1}=\{1,2,3\}$ and $\mathcal{X}_{2}=\{4,5,6,7\}$, that are internally strongly connected, and $\mathcal{S}_{\Gamma}=\mathcal{X}_{1} \cup \mathcal{X}_{2}$. The set $\mathcal{V} \backslash \mathcal{S}_{\Gamma}=$ $\{8,9,10\}$ contains the elements that are not included in any authority. There is always a path from nodes in $\mathcal{V} \backslash \mathcal{S}_{\Gamma}$ to a node in $\mathcal{S}_{\Gamma}$, accordingly to Lemma 3 .

\section{B. The Laplacian Matrix Kernel}

Let us consider a graph of $n$ nodes which is not strongly connected. Suppose there are $m$ authorities $\mathcal{X}_{1}, \ldots, \mathcal{X}_{m}$, each composed of $n_{i}$ nodes, $i=1, \ldots, m$. Thus, $n_{a}=\sum_{i=1}^{m} n_{i}$, and $n=n_{a}+n_{0}$, where $n_{0}$ are the nodes that do not belong to any authority, i.e. $n_{0}=\left|\mathcal{V} \backslash \mathcal{S}_{\Gamma}\right|$. We order the nodes so that the Laplacian is structured as

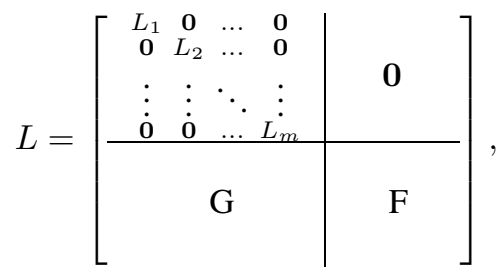

where $L_{1}, \ldots, L_{m}$ are the Laplacian matrices of the authorities subgraphs. The matrix $G$ describes the edges from the nodes in $\mathcal{V} \backslash \mathcal{S}_{\Gamma}$ to the nodes on $\mathcal{S}_{\Gamma}$. Furthermore, $F=$ $L_{\mathcal{V} \backslash \mathcal{S}_{\Gamma}}+H$, where $L_{\mathcal{V} \backslash \mathcal{S}_{\Gamma}}$ is the Laplacian of the subgraph induced by $\mathcal{V} \backslash \mathcal{S}_{\Gamma}$ on $\Gamma$, and $H$ is a diagonal matrix that compensate for the rows of $G$ in the Laplacian $L$, i.e. $h_{i i}=-\sum_{j} g_{i j} \geq 0$.

We first need to investigate the structure of the kernel of $L$. Since $L_{1}, \ldots, L_{m}$ are the Laplacian matrices of the authorities, which are strongly connected because of Lemma 2 , $\operatorname{rank}\left(L_{i}\right)=n_{i}-1, i=1, \ldots, m$. Thus, $\operatorname{rank}(L) \leq n-m$, and in detail, $\operatorname{rank}(L)=n-m-f$, where $f=n_{0}-\operatorname{rank}(F)$. We extend now the results on [10, Lemma 13.1.1] for undirected and oriented graphs, to directed, componentwise weakly connected graphs.

Lemma 4: Consider a graph $\Psi$ on $k$ vertices, its Laplacian matrix $L_{\Psi}$, a matrix $H=\operatorname{diag}\left(h_{1}, \ldots, h_{k}\right), h_{i} \geq 0, i=$ $1, \ldots, k$, and $M_{\Psi, H}=L_{\Psi}+H$. Then, $\operatorname{det}\left(M_{\Psi, H}\right) \geq 0$.

Proof: We use induction on $k$. If $k=1, M_{\Psi, H}=h_{1} \geq$ 0 . If $k>1$ let us call $R=\left\{h_{i}, i=1, \ldots, k: h_{i}>0\right\}$, $r=|R|$ and use induction also on $r$. If $r=0$, then $M_{\Psi, H}=L_{\Psi}$, hence $\operatorname{det}\left(M_{\Psi, H}\right)=0$, since Laplacian matrices have an eigenvalue $\lambda=0$. If $r>0$, let $h_{1}>0$, we expand the determinant along the first row by Laplace formula $\operatorname{det}\left(M_{\Psi, H}\right)=\sum_{j=1}^{k} m_{1 j}(-1)^{1+j} \Xi_{1, j}=\left(h_{1}+\right.$ $\left.\psi_{11}\right) \Xi_{1,1}+\sum_{j=2}^{k} m_{1 j}(-1)^{1+j} \Xi_{1, j}$, where $\Xi_{i, j}$ is the $(i, j)$ minor, the determinant of the matrix obtained from $M_{\Psi, H}$ by removing the row $i$ and the column $j$. Thus, by separating only the term $h_{1} \Xi_{1,1}$

$$
\operatorname{det}\left(M_{\Psi, H}\right)=h_{1} \cdot \operatorname{det}\left(M_{1}\right)+\operatorname{det}\left(M_{2}\right),
$$

where $M_{2}=L_{\Psi}+\operatorname{diag}\left(0, h_{2}, \ldots, h_{k}\right)$, in which the number of elements $h_{i}>0$ is reduced by 1 , enabling induction on $r$. In (6), $M_{1}=L_{\Psi, 1}+H_{1}$, where $L_{\Psi, 1}$ is the Laplacian matrix of the graph $\Psi_{1}$ obtained from $\Psi$ by removing vertex $v_{1}$. We obtain $M_{1}$ from $M_{\Psi, H}$ by removing the first row and the first column, hence its dimension is reduced by 1 , enabling induction on $k$. As a consequence, $H_{1}=\operatorname{diag}\left(h_{2}^{1}, \ldots, h_{k}^{1}\right)$, where $h_{j}^{1}=h_{j}+a_{j 1} \geq 0$ and $a_{j 1}$ is the element at row $i$ and column 1 in the incidence matrix of $\Psi$. By induction hypothesis on $r$, $\operatorname{det}\left(M_{2}\right) \geq 0$, while by the induction hypothesis on $k, \operatorname{det}\left(M_{1}\right) \geq 0$. Thus, $\operatorname{det}\left(M_{\Psi, H}\right) \geq 0$.

Lemma 5: Consider the assumption of Lemma 4, and assume that for each authority $\mathcal{X}_{i}$ of $\Psi$ there exists at least one node $v_{j} \in \mathcal{X}$ such that $h_{j}>0$. Then, $\operatorname{det}\left(M_{\Psi, H}\right)>0$.

Proof: We repeat the same induction arguments of the proof of Lemma 4 . If $k=1, M_{\Psi, H}$ is a positive scalar. For the case $k>1$, consider the authority $\mathcal{X}_{1}$ of $\Psi$ and assume 
the vertex $v_{1} \in \mathcal{X}_{1}$ is such that $h_{1}>0$. Once again,

$$
\operatorname{det}\left(M_{\Psi, H}\right)=h_{1} \cdot \operatorname{det}\left(M_{1}\right)+\operatorname{det}\left(M_{2}\right),
$$

where $M_{1}$ and $M_{2}$ are the same as in Lemma 4, and by the result of Lemma 4 , $\operatorname{det}\left(M_{2}\right) \geq 0$. We prove now that $\operatorname{det}\left(M_{1}\right)>0$, and that the induction hypothesis holds. We need to show that for any authority $\mathcal{Y}$ in $\Psi_{1}$, there exists $v_{j} \in \mathcal{Y}$ such that $h_{j}^{1}>0$.

Let $\mathcal{X}_{1}$ be the authority of $\Psi$ such that $v_{1} \in \mathcal{X}_{1}$. Then, all the authorities of $\Psi$ different from $\mathcal{X}_{1}$ are authorities of $\Psi_{1}$ too. One of the following occurs: $(i) \mathcal{Y}$ coincides with an authority of $\Psi ;(i i) \mathcal{Y} \cap \mathcal{X}_{1} \neq \emptyset ;($ iii $) \mathcal{Y}$ is disjoint from any authority of $\Psi$. In case $(i)$ we have the existence of $v_{j} \in \mathcal{Y}$ such that $h_{j}>0$ by hypothesis. In case $(i i)$, pick a vertex $y_{0} \in \mathcal{Y} \cap \mathcal{X}_{1}$. As $\mathcal{Y}$ is strongly connected in $\Psi_{1}$ (Lemma 2), for every vertex $y \in \mathcal{Y}$ there is a path $\mathcal{P}$ of $\Psi_{1}$ from $y_{0}$ to $y$. However, $y_{0} \in \mathcal{X}_{1}$ and the latter is stable in $\Psi$, hence $\mathcal{P} \subseteq \mathcal{X}_{1}$, and in particular, $y \in \mathcal{X}_{1}$. Thus, $\mathcal{Y} \subset \mathcal{X}_{1}$. Conversely, again by Lemma 2 applied now to $\mathcal{X}_{1}$, for any $v \in \mathcal{Y} \subset \mathcal{X}_{1}$ there exists a path from $v$ to $v_{1}$. Since $\mathcal{Y}$ is stable in $\Psi_{1}$, all the vertices of $\mathcal{P}$ stay in $\mathcal{Y}$ but the last, that goes from $v_{j} \in \mathcal{Y}$ to $v_{1}$. Thus, $a_{j 1}>0$, and $h_{j}^{1}>0$. In case (iii), by Lemma 3 there exists a path $\mathcal{P}$ from $v \in \mathcal{Y}$ to an authority $\mathcal{X}$ of $\Psi$. Since $\mathcal{Y}$ is stable in $\Psi_{1}$, all the edges in $\Psi_{1}$ starting from vertices of $\mathcal{Y}$ lead to vertices in $\mathcal{Y}$. Thus, $\mathcal{P}$ reaches $\mathcal{X}$ passing through $v_{1}$, and again $h_{j}^{1}>0$.

Theorem 1: For any componentwise weakly connected graph and for fixed agreement values $\mu_{1}, \ldots, \mu_{m}$ of the authorities $\mathcal{X}_{1}, \ldots, \mathcal{X}_{m}$, there always exists a single equilibrium point $x=$ $\left[\mu_{1} \ldots \mu_{1} \mu_{2} \ldots \mu_{2} \ldots \mu_{m} \ldots \mu_{m} x_{n_{a}+1} \ldots x_{n_{a}+n_{0}}\right]^{T} \quad$ such that $L x=\mathbf{0}$.

Proof: Let us consider the general form of the Laplacian matrix (5), where $L_{1}, \ldots, L_{m}$ are the Laplacian matrices of the authorities $\mathcal{X}_{1}, \ldots \mathcal{X}_{m}$ and call $\nu^{i}$ the vector

$$
\nu_{j}^{i}=\left\{\begin{array}{lll}
1 & \text { if } & v_{j} \in \mathcal{X}_{i} \\
0 & \text { if } & v_{j} \notin \mathcal{X}_{i}
\end{array}\right.
$$

Since the authorities are not influenced by any external node, $x \in \operatorname{ker}(L)$ can be expressed as

$$
x=z+\sum_{i=1}^{m} \mu_{i} \nu^{i}, \quad \mu_{i} \geq 0, i=1, \ldots, m,
$$

where $z$ is a vector such that $z_{j}=0, \forall j: v_{j} \in \mathcal{S}_{\Gamma}$. Let us call $w=\sum_{i=1}^{m} \mu_{i} \nu^{i}$, then $x \in \operatorname{ker}(L)$ implies

$$
F z=-G w
$$

We prove the non-singularity of $F$ by showing that the assumptions of Lemma 5 are satisfied. Let $\Psi$ be the graph induced by $\mathcal{V} \backslash \mathcal{S}_{\Gamma}$ on $\Gamma$. Then, $F=L_{\Psi}+H$, where $L_{\Psi}$ is the Laplacian of $\Psi$, and $H$ is the diagonal matrix that contributes the rows of $G, h_{i i}=-\sum_{j} g_{i j}$. Following the same reasoning of Lemma (5), for any authority $\mathcal{Y}$ of $\Psi$ there exists $v \in \mathcal{Y}$, connected to a vertex in $\mathcal{S}_{\Gamma}$, hence there exists at least one row of $G$ that gives a contribution $h_{i i}>0$ to $F$. The non-singularity of $F$ follows from Lemma 5 .
Since $F$ is nonsingular, $z=-F^{-1} G w$, exists and is unique, for any fixed values $\mu_{1}, \ldots, \mu_{m}$.

Corollary 1: If the assumptions of Theorem 1 hold and $\mu_{1}=\ldots=\mu_{m}=\mu$, the unique equilibrium of $L x$ is the global consensus $x=\mu \cdot \mathbf{1}$.

Proof: The matrix $\left[\begin{array}{ll}G & F\end{array}\right]$ is constituted by rows of a Laplacian matrix, hence the rows sum to 0 . Thus, $z+w=$ $\mu \cdot \mathbf{1}$ is a solution of $L x=\mathbf{0}$, and Theorem 1 ensures it is the only one for $\mu_{1}=\ldots=\mu_{m}=\mu$, fixed.

Remark 1: The connectivity of the network can be analyzed by the matrices $F$ and $G$. If $F$ and $G$ can be arranged so that $[G \mid F]=\left[\begin{array}{cccc}G_{1} & F_{1} & \cdots & 0 \\ \vdots & \vdots & \ddots & \vdots \\ \dot{G}_{s} & \mathbf{0} & \cdots & \dot{F}_{s}\end{array}\right]$, the network induced on $\Gamma$ by $\mathcal{V} \backslash \mathcal{S}_{\Gamma}$ is constituted by $s$ connected components. The $i^{\text {th }}$ connected component of the induced graph depends on the authority $\mathcal{X}_{j}$ if and only if there is at least one non-zero element in the rows of $G_{i}$ relative to $\mathcal{X}_{j}$. We do not require the network induced on $\Gamma$ by $\mathcal{V} \backslash \mathcal{S}_{\Gamma}$ to be connected, hence $\Gamma$ can be formed by many weakly connected components. The properties stated in this paper are independent from having only one, or many weakly connected components.

\section{Agent Dynamics in Componentwise Weakly Connected CONSEnsus Networks}

We use the results of Section III-B to analyze the asymptotic convergence of the consensus protocol on componentwise weakly connected networks.

Consider a network whose Laplacian matrix is structured as (5). Let $\mathcal{X}_{1}, \ldots, \mathcal{X}_{m}$ be the authorities, and $L_{1}, \ldots, L_{m}$ the corresponding Laplacian matrices, respectively. Partition the full state vector of the system $x$ as

$$
x=\left[\begin{array}{c}
x^{1} \\
\vdots \\
x^{m} \\
x^{0}
\end{array}\right]
$$

where $x^{1} \in \mathbb{R}^{n_{1}}, \ldots, x^{m} \in \mathbb{R}^{n_{m}}$ are the state vectors of the agents in the authorities $\mathcal{X}_{1}, \ldots, \mathcal{X}_{m}$, respectively, and $x^{0} \in \mathbb{R}^{n_{0}}$ is the state vector of the agents in $\mathcal{V} \backslash \mathcal{S}_{\Gamma}$. Due to the structure of the generalized Laplacian matrix (5), the full system dynamics can be decomposed into subsystems. In detail, the authorities are not influenced by any external agent, while the agents in $\mathcal{V} \backslash \mathcal{S}_{\Gamma}$ are influenced by the external agents through the matrix $G$. Thus, the full dynamics can be written as

$$
\begin{aligned}
\dot{x}^{i}(t) & =-L_{i} x^{i}(t), \quad i=1, \ldots, m, \\
\dot{x}^{0}(t) & =-F x^{0}(t)-G\left[\begin{array}{c}
x^{1}(t) \\
\vdots \\
x^{m}(t)
\end{array}\right] .
\end{aligned}
$$

A similar expression can be generated for the discrete-time dynamics

$$
\begin{aligned}
x^{i}(t+1) & =\left(I-\varepsilon L_{i}\right) x^{i}(t), \quad i=1, \ldots, m, \\
x^{0}(t+1) & =(I-\varepsilon F) x^{0}(t)-G\left[\begin{array}{c}
x^{1}(t) \\
\vdots \\
x^{m}(t)
\end{array}\right] .
\end{aligned}
$$


Theorem 2: Given any initial agent state vector $x(0)$ the consensus protocol on a componentwise weakly connected network with Laplacian matrix (5) converges to a value $\bar{x}$, where $\bar{x}^{1}=\mu_{1} \mathbf{1}, \ldots, \bar{x}^{m}=\mu_{m} \mathbf{1}$ are consensuses among the agents in the authorities $\mathcal{X}_{1}, \ldots, \mathcal{X}_{m}$, respectively, and $\bar{x}^{0}=-F^{-1} G\left[\begin{array}{c}\bar{x}^{1} \\ \vdots \\ \bar{x}^{m}\end{array}\right]$, independently from $x^{0}(0)$.

Proof: The dynamics of the authorities are described by standard consensus, hence the authorities states will asymptotically converge. Let $\lim _{t} x^{i}(t)=\bar{x}^{i}=\mu_{i} \mathbf{1}$. The dynamics of the agents in $\mathcal{V} \backslash \mathcal{S}_{\Gamma}$ are described by (10b) ((11b) for the discrete-time case). For $t \rightarrow \infty$ these can be regarded as the dynamics of a linear system subject to a constant input

$$
\dot{x}^{0}=A x^{0}+B \bar{u}
$$

where $\bar{u}=\left[\begin{array}{c}\bar{x}^{1} \\ \vdots \\ \bar{x}^{m}\end{array}\right] \in \mathbb{R}^{n_{a}}$. The Gershgorin's theorem ensures that all the eigenvalues of $A$ are in the half plane $\operatorname{Re}(\lambda) \leq 0$, and Lemma (5) and Theorem (1) ensure that none of the eigenvalues is 0 . Thus, $A$ is stable, and the system state asymptotically converges to

$$
\bar{x}^{0}=-A^{-1} B \bar{u}
$$

for continuous-time dynamics. Stability is proven similarly for the discrete-time case, where the state converges to

$$
\bar{x}^{0}=(I-A)^{-1} B \bar{u} .
$$

Both (13) and (14) can be expressed in terms of the $F$ and $G$ matrices in (5)

$$
\bar{x}^{0}=-F^{-1} G \bar{u}=\Phi \bar{u},
$$

where $\Phi$ is the dc-gain of the subsystem (12), whose state vector is constituted by the states of the agents in $\mathcal{V} \backslash \mathcal{S}_{\Gamma}$.

Corollary 2: If $\bar{x}^{1}=\mu 1, \ldots, \bar{x}^{m}=\mu 1$ then the network converges to the consensus $\bar{x}=\mu \mathbf{1}$, which is independent from the value of $x^{0}(0)$.

Proof: Since the dynamics of the agents in $\mathcal{V} \backslash \mathcal{S}_{\Gamma}$ are described by a linear stable system forced by constant input, the initial state $x^{0}(0)$ does not affect the asymptotic value $\bar{x}^{0}$. Due to equation (9) and to Corollary 1 , for $\bar{u}=\mu \mathbf{1}$, $-F^{-1} G \bar{u}=\mu 1$. Hence, when the different authorities are in consensus, the agents that are not included in any authority agree on such a consensus. As a consequence of (9), we also have that given $\Phi, \sum_{j=1}^{n_{a}} \phi_{i j}=1, i=1, \ldots, n_{0}$.

The result of Corollary 2 ensures that if there is a single authority $\mathcal{X}_{1}$, whose agents agree on $\mu_{1}$, the whole network agrees on $\mu_{1}$, as mentioned in [8]. Note that by Theorem 1 the agents in $\mathcal{V} \backslash \mathcal{S}_{\Gamma}$ does not need to be (and in general will not be) in agreement.

We give now a result on the robustness of strongly consensus networks in which some links break, in a way that the network becomes componentwise weakly connected. In particular we prove that if the difference between the consensuses reached by the authorities is small, the asymptotic values reached by the agents that do not belong to any authorities are close to each others, and close to the ones of the authorities.

Theorem 3: Assume $\mu_{1} \leq \ldots \leq \mu_{m}$ be the consensuses of the authorities $\mathcal{X}_{1}, \ldots, \mathcal{X}_{m}$, then $\mu_{1} \leq \bar{x}_{i}^{0} \leq \mu_{m}, i=$ $1, \ldots, n_{0}$.

Proof: Let us assume $\min _{i} \bar{x}_{i}^{0}=\underline{\mu}<$ $\min \left(\mu_{1}, \ldots, \mu_{m}\right)$, and subtract from each component of $\bar{x}$ the value $\mu$, so that $\bar{x} \geq \mathbf{0}$, and $\bar{x}^{k}>\mathbf{0}, k=1, \ldots, m$. We show that this is impossible.

Let $\mathcal{J}=\left\{i \in\left[1, n_{0}\right]: x_{i}^{0}=0\right\}$ and arbitrarily select $\bar{\imath} \in \mathcal{J}$. Consider the $\bar{t}^{t h}$ row of equation (9), we have $\sum_{j=1}^{n_{0}} f_{\bar{\imath} j} \bar{x}_{j}^{0}+\sum_{j=1}^{n-n_{0}} g_{\bar{\imath} j} \bar{x}_{j}=0$, where all the coefficients $f_{\bar{\imath} j}$ and $g_{\bar{\imath} j}$ are non-positive, except $f_{\bar{\imath}}$. However, since $\bar{x}_{\bar{\imath}}^{0}=0$ the term relative to $f_{\bar{\imath}}$ does not contribute, and since $\bar{x}_{j}>0, j=1, \ldots, n-n_{0}$, also $g_{\bar{\imath} j}=0, j=1, \ldots, n-n_{0}$. Even the terms $f_{\bar{\imath} j} \bar{x}_{j}^{0}, j=1, \ldots, n_{0}, j \neq \bar{\imath}$ must be zero, and hence either $f_{\bar{\imath} j}=0$, which means that there is no edge $e_{\bar{\imath} j}$, or $\bar{x}_{j}^{0}=0$, namely $j \in \mathcal{J}$. This would imply $\mathcal{J}$ to be a stable subgraph of $\Gamma$, which is impossible because any stable subgraph contains a minimal stable set, that is an authority, and by Definition $4, \mathcal{V} \backslash \mathcal{S}_{\Gamma}$ contains the nodes that are not included in any authority. Hence, the minimum should be attained at a node in $\mathcal{S}_{\Gamma}$.

The same reasoning can be repeated to show that $\bar{x}_{i}^{0} \leq$ $\max _{j=1, \ldots n-n_{0}} \bar{x}_{j}, i=1, \ldots, n_{0}$, by a similar impossibility argument on $\max _{i} \bar{x}_{i}^{0}=\bar{\mu}>\max \left(\mu_{1}, \ldots, \mu_{m}\right)$.

The result of Theorem 3 indicates that if the consensus values of the different authorities are close, also the asymptotic values of the agents in $\mathcal{V} \backslash \mathcal{S}_{\Gamma}$ will be close to such values, no matter what is the initial state of the agents. Thus, if the agents are measuring a quantity with uniform distribution, one may expect that in case of link failures that generate a componentwise weakly connected topology, the estimate is still valid. In fact, each of the authorities will perform a local estimate, and all of them should be close to each others because of the uniform distribution of the estimated quantity. The agents that does not belong to any authority will stay close to such values. On the other hand, it may be critical to apply a consensus protocol subject to link interruptions to detect local phenomena, because if these happen in a region covered by nodes in $\mathcal{V} \backslash \mathcal{S}_{\Gamma}$, they will be completely discarded.

We give an additional result that help in localizing the values of $\bar{x}_{i}^{0}, i=1, \ldots, n_{0}$, given $\mu_{j}, j=1, \ldots, n_{a}$.

Corollary 3: Function (15) is nondecreasing from any $\bar{u}_{j}, j=1, \ldots n_{a}$ to any $x_{i}^{0}, i=1, \ldots n_{0}$, and $\bar{x}_{i}^{0}, i=$ $1, \ldots, n_{0}$ is in the convex combination of $\mu_{1}, \ldots, \mu_{m}$.

Proof: Assume $\bar{u}^{(1)} \geq \bar{u}^{(2)}$, we prove that for the corresponding values $\bar{x}^{0,(1)} \geq \bar{x}^{0,(2)}$. By linearity $\Phi\left(\bar{u}^{(1)}-\right.$ $\left.\bar{u}^{(2)}\right)=\bar{x}^{0,(1)}-\bar{x}^{0,(2)}$, and since $\bar{u}^{(1)}-\bar{u}^{(2)} \geq \mathbf{0}$, by the result of Corollary $3, \bar{x}^{0,(1)}-\bar{x}^{0,(2)} \geq \mathbf{0}$, which proves nondecreaseness. By Corollary (1) we have that $\sum_{j} \phi_{i j}=1$, $i=1, \ldots, n_{0}$, and since $\Phi$ is the matrix of a non-decreasing function, $\phi_{i j} \geq 0, i=1, \ldots, n_{0}, j=1, \ldots, n_{a}$. Thus, calling $\eta_{k}=\sum_{h=1}^{k} n_{i}$ and $\eta_{0}=0, \bar{x}_{i}^{0}=\sum_{j} \phi_{i j} \bar{u}_{j}=$ $\sum_{k=1}^{m}\left(\sum_{j=1+\eta_{k-1}}^{\eta_{k}} \phi_{i j}\right) \mu_{k}$, that is the definition of convex 


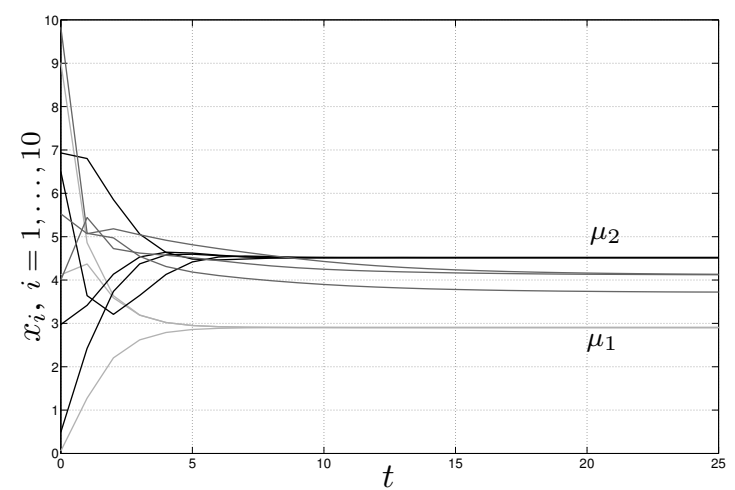

Fig. 2. Simulation of the dynamics of the weakly connected consensus network shown in Figure 1. The authorities $\mathcal{X}_{1}$ and $\mathcal{X}_{2}$ reach two different agreements and the other nodes settle in the middle.

combination, because of the properties of the matrix $\Phi$.

The matrix $\Phi$ being composed of nonnegative elements implies that if the agreement of one of the authorities increases, the asymptotic state values of the agents in $\mathcal{V} \backslash \mathcal{S}_{\Gamma}$ do not decrease. More in details we note that if the network is weakly connected, the function represented by $\Phi$ is monotonically increasing from any input to any output. In fact, assume that the agreement value of an authority -say $\mathcal{X}_{1}$ - increases, and the value of a node $v_{i} \in \mathcal{V} \backslash \mathcal{S}_{\Gamma}$ remains unchanged. This is possible only if there is no directed path from $v_{i}$ to $\mathcal{X}_{1}$, but since $\mathcal{X}_{1}$ is stable and does not have any outgoing edges, there is not even an undirected path. Hence, the network must be disconnected.

\section{Simulations}

We present now simulations that provide an experimental proof of the results presented in Sections III and IV. We consider the weakly connected network shown in Figure 1, where the dashed links are removed and all the edges are weighted 1. The Laplacian matrix of such a network is in the form (5), where $m=2$ and $L_{1}\left[\begin{array}{rrr}2 & -1 & -1 \\ -1 & 2 & -1 \\ -1 & 0 & 1\end{array}\right], L_{2}=\left[\begin{array}{rrrr}2 & -1 & -1 & 0 \\ 0 & 1 & -1 & 0 \\ 0 & 0 & 1 & -1 \\ -1 & -1 & 0 & 2\end{array}\right], F=\left[\begin{array}{rrr}3 & -1 & -1 \\ 0 & 1 & -1 \\ -1 & 0 & 2\end{array}\right]$, $G=\left[\begin{array}{rrrrrrr}-1 & 0 & 0 & 0 & 0 & 0 & 0 \\ 0 & 0 & 0 & 0 & 0 & 0 & 0 \\ 0 & 0 & 0 & -1 & 0 & 0 & 0\end{array}\right]$. We set the initial condition of each agent to a random number uniformly distributed in $[0,10]$ and we run the consensus protocol (2) with agents dynamics (3), where $\varepsilon=0.3$. As shown in Figure 2 and accordingly to Theorem 2 , the nodes in $\mathcal{X}_{1}$ (light gray) converge to an agreement on value $\mu_{1}$, the nodes in $\mathcal{X}_{2}$ (black) converges to a different agreement on value $\mu_{2}$, while the remaining nodes(dark gray) converge to values in the middle. This is also consistent with Theorem 3, and the dc-

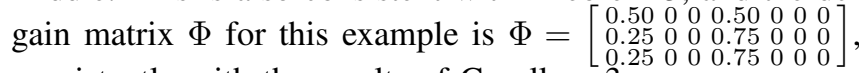
consistently with the results of Corollary 3 .

In Figure 3 we plot the trajectory in the phase plane $\left(x_{8}, x_{9}\right)$ for different initial conditions $\left(x_{8}(0), x_{9}(0)\right)=$ $(i, j), i, j=1, \ldots, 10$. The final value indicated by the cross is always the same, since accordingly to Theorem 2 the initial conditions of the agents in $\mathcal{V} \backslash \mathcal{S}_{\Gamma}$ does not affect the final value. By setting $x^{1}(0)=\left[\begin{array}{lll}4 & 7.5 & 3.2\end{array}\right]^{T}$ and

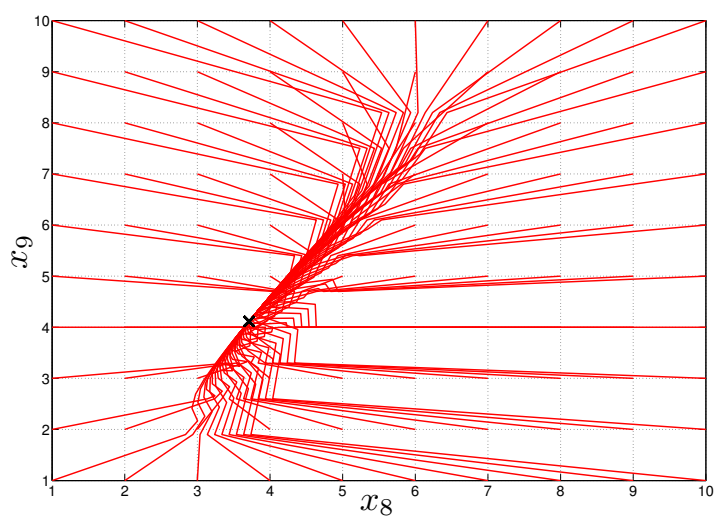

Fig. 3. Trajectories in the phase plane section $\left(x_{8}, x_{9}\right)$ for different initial conditions $\left(x_{8}(0), x_{9}(0)\right)$.

$x^{2}(0)=\left[\begin{array}{llll}22 / 6 & 5.2 & 4.5 & 2.2\end{array}\right]^{T}$, the two authorities converges to $\mu_{1}=\mu_{2}=4.1833$, accordingly to the result in [4, Corollary 2]. Independently from the initial states of the remaining agents, the whole network reaches a consensus on $\mu_{1}=\mu_{2}$, consistently with Corollary 2 .

\section{CONCLUSIONS}

We have presented an analysis of the dynamic consensus protocol for the case in which the graph defining the network topology is componentwise weakly connected. We have provided the general result that ensures that the agents dynamics converge, we have provided conditions on the possibility of reaching an agreement between all the nodes, and we have given bounds on the residual disagreement for the other cases. An application of these results for links failures detection and repairing is currently under study.

\section{REFERENCES}

[1] N. A. Lynch, Distributed Algorithms. San Mateo, CA: Morgan Kaufmann, 1997.

[2] K. M. Chandy and M. Charpentier, "Self-similar algorithms for dynamic distributed systems," in International Conference on Distributed Computing Systems, Toronto, Canada, 2007, p. 67.

[3] L. Xiao and S. Boyd, "Fast linear iterations for distributed averaging," Systems and Control Letters, vol. 53, no. 1, pp. 65-78, Sept. 2004

[4] R. Olfati-Saber and R. M. Murray, "Consensus problems in networks of agents with switching topology and time-delays," IEEE Trans. Automatic Control, vol. 49, no. 9, pp. 1520-1533, Sept. 2004.

[5] D. Bauso, L. Giarre, and R. Pesenti, "Nonlinear protocols for optimal distributed consensus in networks of dynamic agents," Systems and Control Letters, vol. 55, no. 11, pp. 915-928, Nov. 2006.

[6] W. Ren, R. W. Beard, and E. M. Atkins, "A survey of consensus problems in multi-agent coordination," in American Control Conference, Portland, OR, 2005, pp. 1859-1864.

[7] R. Olfati-Saber, "Distributed kalman filter with embedded consensus filters," in Proc. 44th IEEE Conf. on Decision and Control, Seville, Spain, 2005, pp. 8179-8184.

[8] V. Gupta, C. Langbort, and R. M. Murray, "On the robustness of distributed algorithms," in Proc. 45th IEEE Conf. on Decision and Control, San Diego, CA, 2006, pp. 3473-3478.

[9] R. Merris, "Laplacian matrices of a graph: a survey," Linear Algebra and its Application, vol. 197, pp. 143-176, 1994.

[10] C. Godsil and G. Royle, Algebraic Graph Theory, ser. Graduate Texts in Mathematics. New York: Springer-Verlag, 2001, vol. 207.

[11] R. Diestel, Graph Theory, ser. graduate texts in mathematics. New York: Springer-Verlag, 2000, vol. 173. 